\title{
De quelques araires au Népal. Tentative de classification par les contraintes
}

of ploughs (ards) in Nepal. Attempt of classification through constraints

Acerca de algunos arados en Nepal. Tentativa de clasificación por las

restricciones

Denis Blamont

\section{OpenEdition}

Journals

Édition électronique

URL : https://journals.openedition.org/tc/256

DOI : $10.4000 /$ tc. 256

ISSN : 1952-420X

Éditeur

Éditions de l'EHESS

Édition imprimée

Date de publication : 1 juillet 2001

Pagination : $51-76$

ISSN : 0248-6016

\section{Référence électronique}

Denis Blamont, « De quelques araires au Népal. Tentative de classification par les contraintes »,

Techniques \& Culture [En ligne], 37 | 2001, mis en ligne le 20 octobre 2005, consulté le 29 septembre 2022. URL : http://journals.openedition.org/tc/256 ; DOI : https://doi.org/10.4000/tc.256

Ce document a été généré automatiquement le 29 septembre 2022.

Tous droits réservés 


\title{
De quelques araires au Népal. Tentative de classification par les contraintes
}

\author{
Of ploughs (ards) in Nepal. Attempt of classification through constraints \\ Acerca de algunos arados en Nepal. Tentativa de clasificación por las \\ restricciones
}

Denis Blamont

1 L'étude locale des formes d'un instrument agricole, et tout particulièrement de l'araire, est toujours délicate en ce que les éléments à prendre en compte sont nombreux, et leurs combinaisons plus nombreuses encore. Le choix de ces éléments et combinaisons pat les agriculteurs est contraint tant par les matériaux disponibles (bois et fer) et les savoir-faire techniques requis pour la fabrication du dispositif que par les circonstances de son utilisation. Les contraintes vont de la nature des sols aux gestes du laboureur, en passant par le climat, la pratique de l'irrigation, les façons agricoles...

2 Au Népal en particulier, où les migrations et les échanges inter- et intra-régionaux sont constants et très nombreux, et où les occasions de voir d'autres outils que les siens et de s'en inspirer ne manquent donc pas, à la diversité des milieux répond une grande variété des araires d'une vallée à l'autre, parfois dans une même vallée ou sur un même versant, voire au sein d'une même exploitation. De plus, les formes très élaborées de ces araires, en dépit d'une apparente simplicité, ainsi que le discours des utilisateurs, justifient qu'on s'essaie à trouver les raisons fonctionnelles de cette variété.

3 Par ailleurs, une classification reprenant uniquement la typologie habituelle, fondée sur la morphologie, qui reste valable à l'échelle mondiale ou même régionale, aboutirait à des redites dès lorsqu'on chercherait à rendre compte de cette diversité et de caractères qui paraissent être des « détails » du seul point de vue de la forme. Surtout, elle placerait des araires très comparables, quant à leurs conditions d'utilisation, dans des groupes très éloignés et rapprocherait abusivement des araires très différents $d u$ point de vue de leurs déterminants fonctionnels. À l'inverse, une description 
fonctionnelle ou uniquement déterministe ne saurait rendre compte de types dissemblables répondant aux mêmes contraintes.

4 Ainsi, s'il faut constater la présence de deux types bien distincts (le dental et le manche-sep2) qui relèvent d'aires culturelles différentes, nous nous proposons d'identifier les traits communs de leurs variantes. On ne retiendra donc la typologie morphologique d'Haudricourt et J.-Brunhes-Delamarre (1986) qu'en l'inversant. C'est-àdire en considérant, d'une part, un type, dit générique dans les ouvrages généraux, tel le «dental» ou le «manche-sep», comme une particularité régionale (historique); d'autre part, des familles d'« états finaux ", hétérogènes quant à ces types, mais qui présentent des traits d'adaptation - plus ou moins récente- comparables, comme constituées par des tendances ${ }^{3}$. En d'autres termes, la classification proposée ici repose bien moins sur la constatation de la présence au Népal de deux grands types génériques d'araires (et de leurs variantes), que sur leurs adaptations à des contraintes similaires.

5 Afin de fonder cette classification, nous nous efforcerons dans un premier temps de recenser ces contraintes et déterminants régionaux ainsi que les réponses qui leur ont été «trouvées $»^{4}$, tout en gardant à l'esprit le fait que leur absence éventuelle n'implique pas forcément un choix des laboureurs, mais peut-être une simple ignorance. Ces faisceaux de contraintes - dont la hiérarchisation varie d'une région à l'autre- et ces réponses nous permettront alors d'envisager les axes d'une typologie recoupant les aires de répartition des grands types morphologiques des classifications habituelles: nous distinguerons ainsi «les araires du Teraï», "les araires des moyennes montagnes » et « les araires des hauts pays ».

\section{Les contraintes}

6 On peut identifier trois grands types de contraintes. Les plus importantes sont bien évidemment induites par l'état et la nature du champ à labourer; viennent ensuite celles qui sont liées aux types de labouts, et enfin celles relatives aux matériaux de fabrication de l'araire. Transversalement, les contraintes qui relèvent des habitudes et des savoir-faire hérités ou acquis viennent parfois relativiser l'adéquation des différentes parties des araires aux deux premiers types de contraintes. Nous ne nous étendrons pas sur le nombre d'animaux de trait. En effet, nous n'avons connaissance à ce jour que d'un exemple au Népal d'attelage à un seul animal, dans le sud Dolpo (cf. Dollfus et al., ce volume); dans les moyennes et basses montagnes, les zébus ne semblent pas assez robustes pour être attelés seuls.

\section{L'état et la nature des champs}

7 De l'état hydrique des sols dépendent la profondeur du labour et la largeur du sillon. Dans les régions de climats peu humides (ouest ${ }^{5}$ ) ou semi-arides (nord), il est nécessaire de labourer peu profond et de bien émietter la terre afin d'en conserver l'humidité ${ }^{6}$. Il convient alors d'avoir un sep aussi large que possible : un sep horizontal permet de bien travailler le sol car il peut être long et s'élargir depuis le soc jusqu'au talon sans être trop difficile à tirer, puisqu'il attaque le sol progressivement, à condition qu'il ne s'y enfonce pas trop profondément. Suivant les régions du Népal, une chambige, deux types de seps et deux types de socs permettent de répondre à ces contraintes. 
8 L'araire dental des moyennes montagnes de l'Extrême Ouest (Jumla, Bajura, Bajhang...) est équipé d'une chambige qui assure l'horizontalité du sep, lui-même droit. S'il faut en croire Haudricourt, la technique des bois recourbés est antérieure à celle des bois droits et l'on a peut-être affaire ici à un araire ancien dont la persistance tient à sa très bonne adaptation au milieu.

Pour ce qui concerne les manche-sep ${ }^{7}$, on en trouve de deux sortes adaptées aux sols secs.

10 . L'araire monobloc des hautes vallées du versant nord de la grande chaine et des régions tibétaines au nord, où l'agriculture n'est possible qu'irriguée. Contrairement au sep courbe du Mustang et du Dolpo (fig. 1; Dollfus et al., ce volume : fig. 2), le sep de l'araire de la vallée de la Tsum (derrière le Ganesh Himal, dans le Népal central) est droit et horizontal, grâce aux angles, très aigus, qu'il fait avec le timon et son manche, comme celui de certains araires des régions tibétaines immédiatement au nord (fig. 1). Ces araires ont des seps très larges flanqués d'ailes qui en augmentent encore la largeur. Le nombre de labours précédant les semailles est souvent assez important (entre deux et trois), que ces labours soient effectués par un seul ou plusieurs attelages.

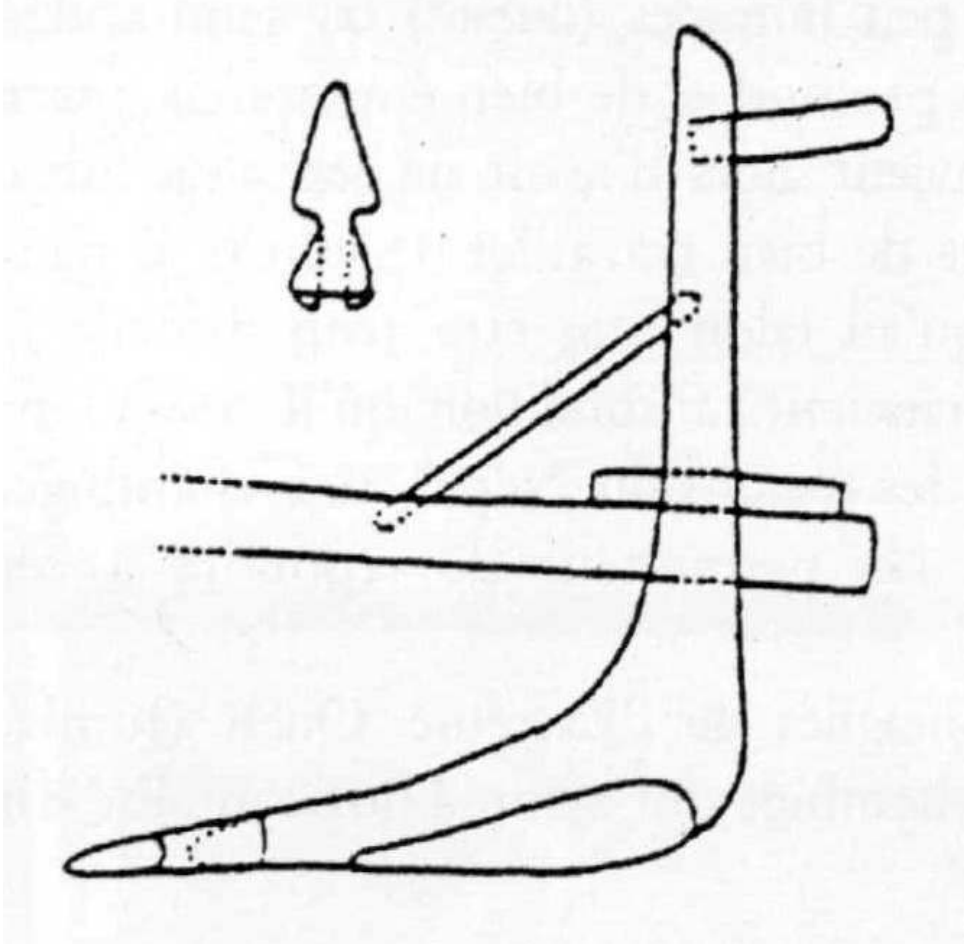

Figure 1. Araire monobloc à genou, larges ailes et à soc à douille en fer de flèche (Tsum)

11 . L'araire à « tête de sep rapportée » du Teraï de l'extrême ouest (photo 1 ; Dollfus et al : fig. 5.3) est similaire à celui qu'on trouve dans les hautes vallées du Kumaon oriental. La tête du sep est fichée par un tenon dans le manche (photo 2), dont l'extrémité forme donc la partie arrière de la semelle, comme au Panjab (Haudricourt 1986 : 261, fig. 116). Sur les sols les plus secs (basse vallée de la Bheri), la tête du sep est plus large et peut même être très longue et envelopper le corps du sep ${ }^{8}$, comme au Kumaon voisin, où il est équipé d'une douille conique, alors qu'au Népal, il est équipé d'une reille ${ }^{9}$ assez courte. 
12 étroits et plus l'angle d'incidence du sep avec le sol est obtus, afin, nous le verrons, d'assurer une pénétration plus profonde. C'est le cas non seulement de l'araire du Teraï central et oriental, que nous appellerons « à mancheron accolé » au sep (photos 3 et 4), mais également des araires des « moyennes montagnes » du centre Népal (régions de la Trisuli, de l'Ankhu Khola et de la Buri Gandaki), ou encore du «manche-sep à tête de sep rapportée » du sud du bassin de Sutkhet, aux sols humides mais assez grossiers. Là, le sep est très étroit et la reille, très longue, traverse le manche (photo 1), comme en Afghanistan du Nord-Est et dans l'Inde du Nord-Ouest. Aucun de ces araires n'est pourvu d'ailes, dont la technique est inconnue.

C'est également à la nécessité de bien émietter le sol qu'on doit attribuer la présence du soc à douille dans les hautes vallées du Népal (mais aussi des régions du Tibet situées plus au nord). La douille, qui protège bien la tête du sep, autorise une large pénétration dans le sol et cela d'autant plus qu'elle est en forme de pointe de flèche ${ }^{10}$ dans des régions de la Tsum, du Haut Mustang et du Dolpo, sur le modèle tibétain (fig. 1).

Quand on descend vers des pays moins secs, la douille perd sa forme de flèche, devient plus conique et fine (fig. $2 \mathrm{~b}$ ) puis, généralement, laisse la place à la reille. Cette dernière permet de tracer des sillons étroits et denses et assure une moindre résistance à la traction dans des sols plus humides et plus lourds.

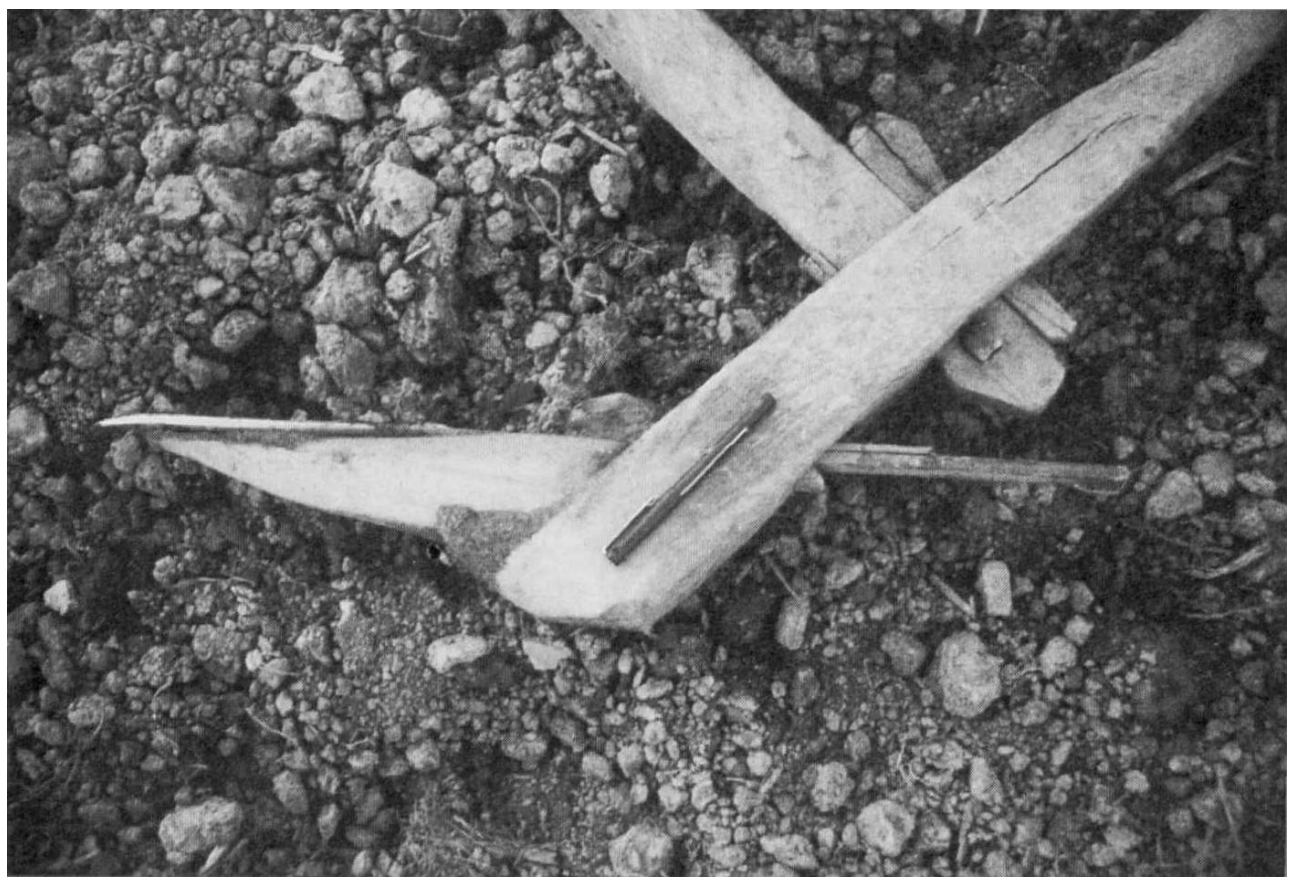

Photo 1. Araire à tête de sep rapportée (Mehalkuna, Bheri inférieure) 


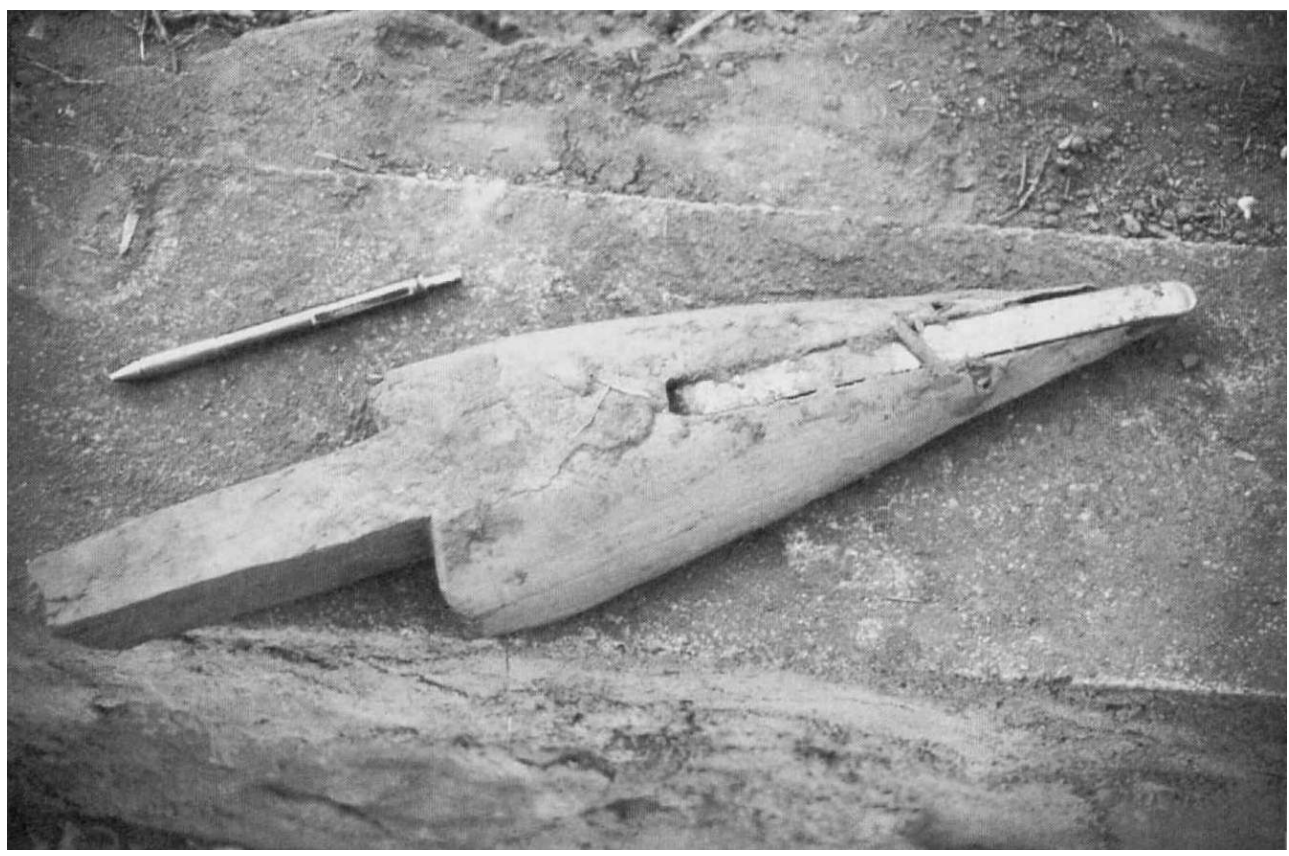

Photo 4. Araire de Letang à long manche et mancheron accolé (Est du Teraï)

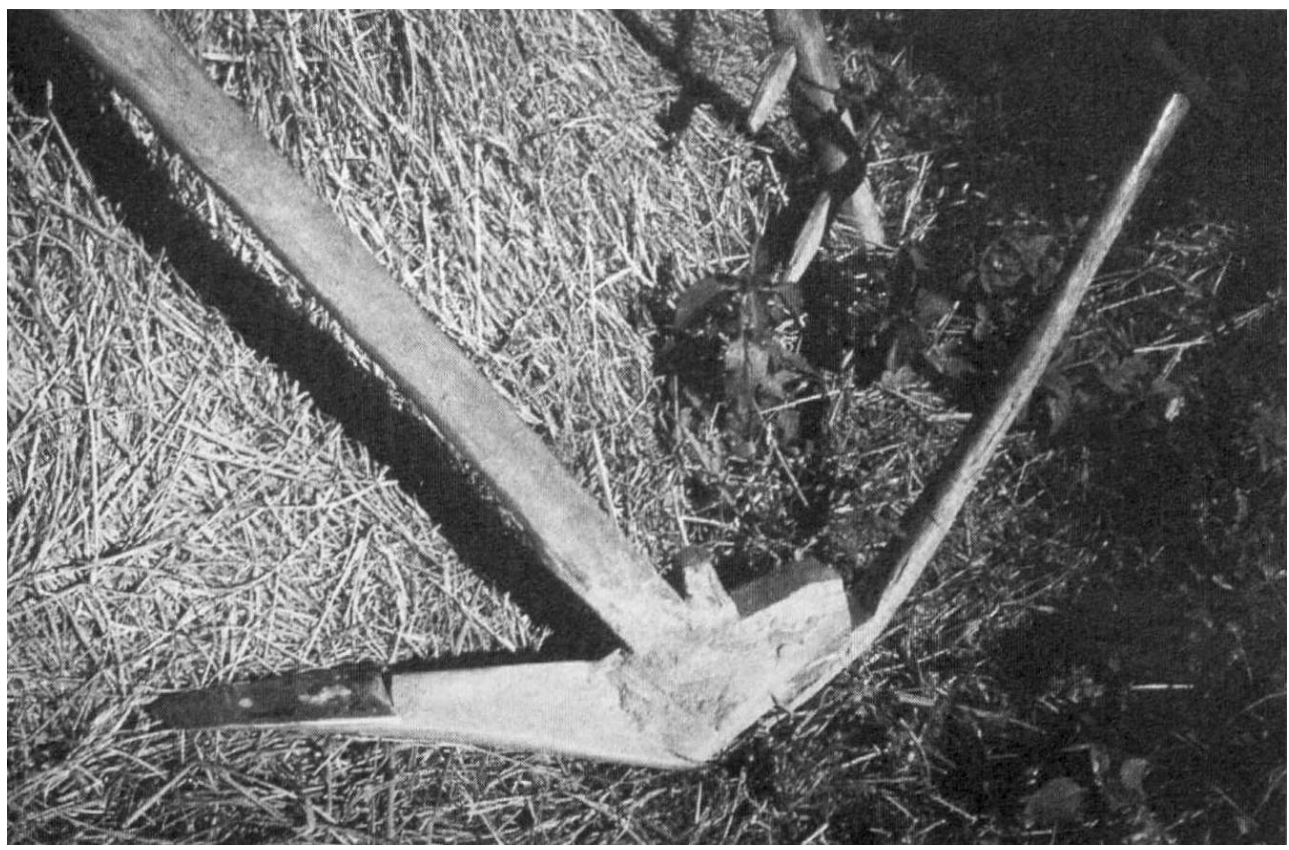

Photo 3. Araire à soc en forme de couteau et mancheron accolé (Janakpur, Est du Teraï) 


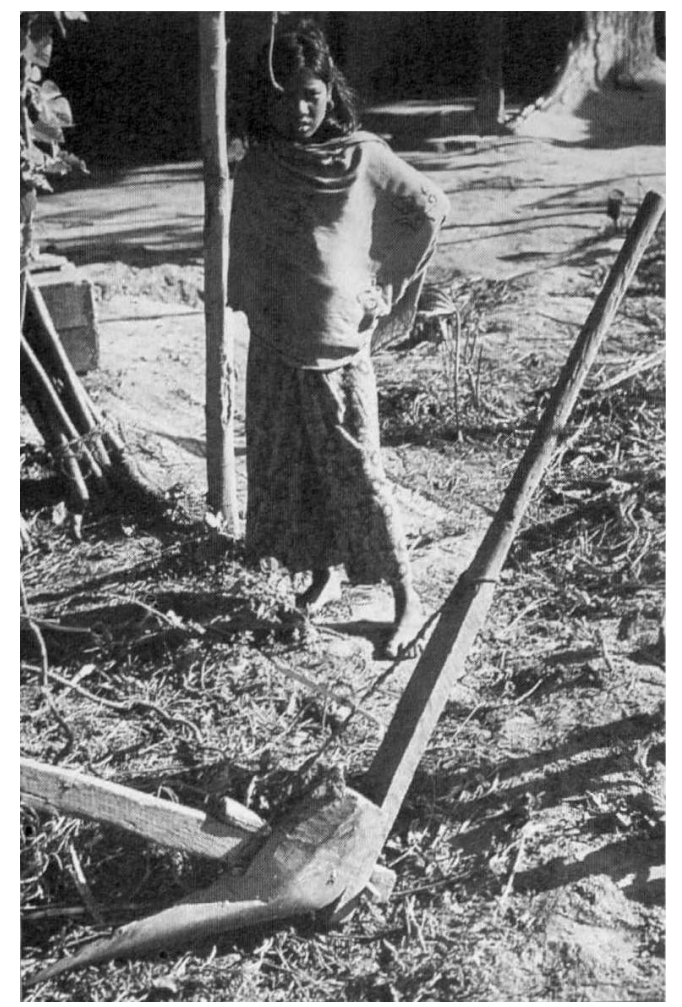

Photo 4. Araire de Letang à long manche et mancheron accolé (Est du Teraï)

Dans les régions tibétaines, ainsi que dans les vallées en auge des hauts pays du versant sud de la grande chaîne, les champs se trouvent le plus souvent sur de vastes étendues sub-horizontales. En revanche, dans les moyennes et basses vallées de ce versant sud, ce sont surtout les pentes qui sont cultivées et leurs sols sont plus grossiers, parsemés d'éboulis et difficiles à travailler. Cette difficulté impose des araires solides, tel le manche-sep monobloc, particulièrement adapté à ce type de sol. On rencontre néanmoins dans les sols grossiers, caillouteux et difficiles à pénétrer des régions sèches, deux contraintes qui s'opposent : la nécessité déjà soulignée d'un sep horizontal et large pour bien émietter la terre ; celle d'un angle d'incidence assez accentué et d'un ensemble soc-sep fin pour y pénétrer. Cette opposition a été résolue de manières différentes suivant les types d'araires.

Dans les moyennes montagnes du Népal central (Dhading, Nuwakot, Rasuwa), le sep perd ses ailes et la douille sa forme de flèche. L'angle d'incidence du sep est assez aigu (fig. 2a). Le timon, raccourci, est relié au joug par une longue lanière qui permet, en agissant sur le manche qu'on fait basculer vers le haut tout en appuyant sur le timon, d'augmenter encore l'angle d'incidence : le laboureur doit alors marcher à côté de son araire et non derrière. Les seps non protégés par des ailes s'usent assez rapidement, et la profondeur du labour dépend à la fois de la longueur et de l'angle d'incidence du sep ; il est donc nécessaire d'en pallier l'usure en faisant varier cet angle à l'aide de cales placées au-dessus et au-dessous du timon, à l'endroit où il traverse le sep. 


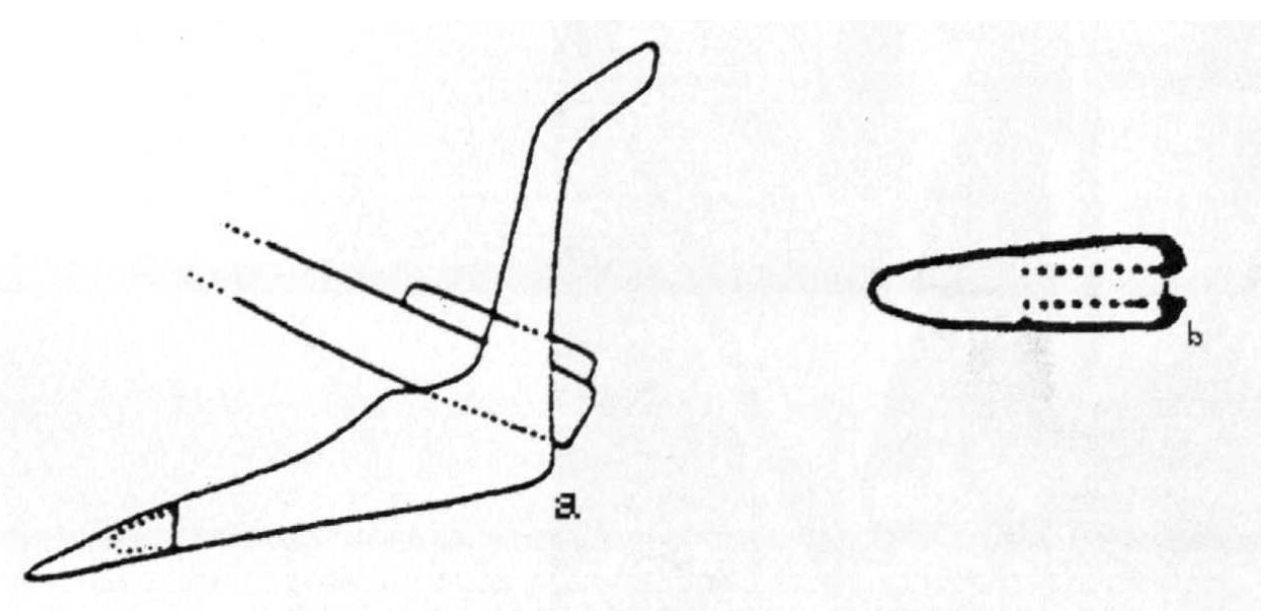

Figure 2. Araire manche-sep (a) à douille conique (b) (Haute Trisuli) derrière le manche où le laboureur peut poser le pied et peser ainsi sur le sep. D'après Haudricourt, c'est un araire « qui pénètre mieux dans les sols pierreux parce qu'on peut faire pression avec le pied sur le $\operatorname{sep}^{11}$ ». Je suis pour ma part assez sceptique sur ce point et j'aurais tendance à penser que cette fonction est assurée plus efficacement par le manche-sep, grâce à la variabilité de l'angle que fait le sep avec le timon. D'où une formulation légèrement différente de celle d'Haudricourt: l'horizontalité du sep du dental me semble être destinée à empêcher une trop grande pénétration dans des sols de régions sèches dont il importe de maintenir l'humidité, et peser du pied sur le sep me paraît avoir pour fonction, non de l'enfoncer, mais bien de l'empêcher de remonter. La pénétration dans le sol en début de raie est obtenue par une pression sur la chambige et une action sur le manche. Signalons ici la supériorité, de ce point de vue, du dental népalais sut celui qu'on trouve dans le Chota Nagpur, au Bihar, sur lequel l'espace où s'exerce la pression du pied se trouve entre le manche et le timon, ce qui oblige alors le laboureur à cheminer à côté de l'araire.

Plus les sols deviennent humides et fins, plus les sillons peuvent être profonds, étroits et denses. On a vu plus haut que les seps devenaient alors de plus en plus étroits et que la reille remplaçait la douille. Le réglage de la profondeur du labour se fait de trois manières (dont la première n'exclut pas l'une des deux autres) : par l'angle que fait le sep avec le timon; par des crans sur le timon, quand il est fixé directement au joug (à l'est et au centre du Teraï, photo 5) ; par la longueur de la lanière qui relie le timon au joug, quand il ne lui est pas directement attaché (centre du Teraï). 


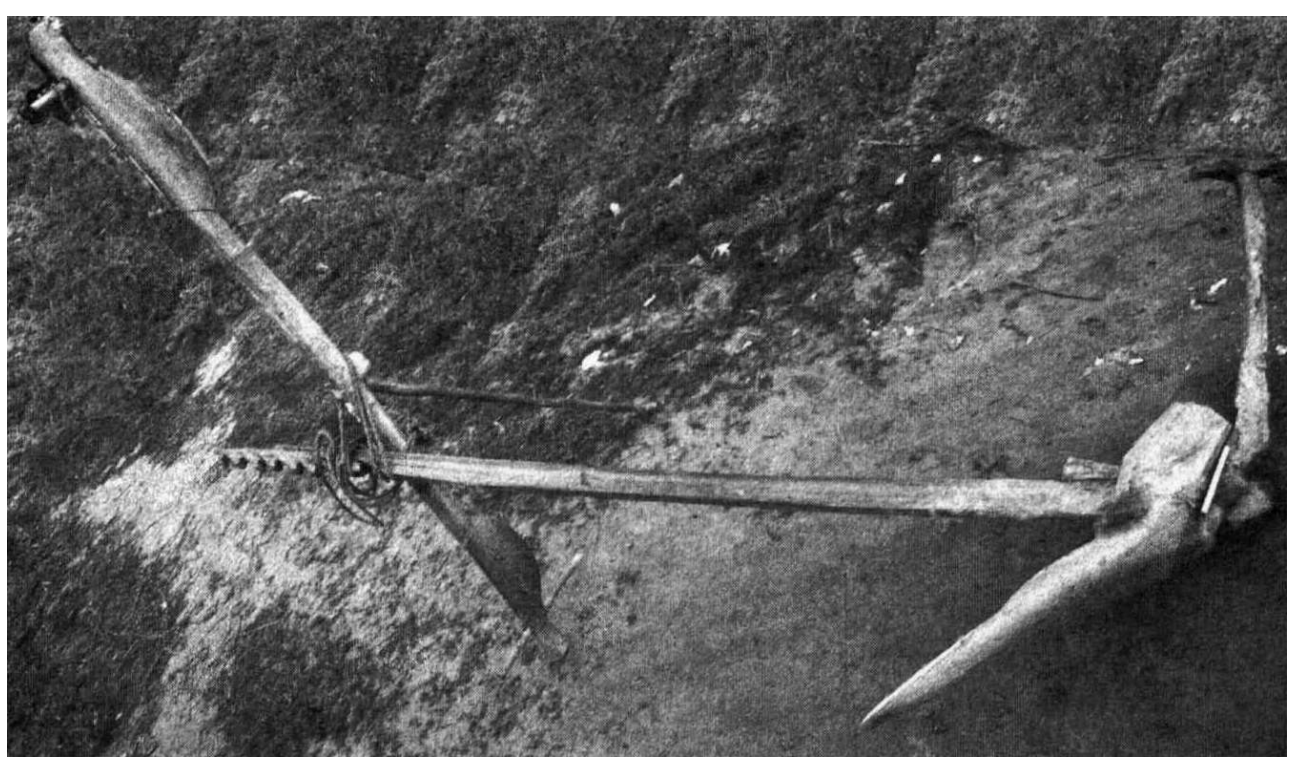

Photo 5. Araire à mancheron accolé et timon cranté (Chitwan, Centre du Teraï) afin de ne pas enfouir les semences trop profondément et de ne pas fatiguer les animaux de labour, surtout dans les sols lourds. C'est ce trait qui me semble rendre compte de la forme de l'araire manche-sep à sep court et à « mancheron accolé », dont la pénétration dans le sol est aisée, car l'angle d'incidence peut être très ouvert, puisque, on l'a vu, le sep n'a pas besoin d'être horizontal : le mancheron n'est pas fixé sur le sep mais derrière, passant même parfois presque dessous (photo 3), et s'avère le plus souvent traversé par le timon qu'il permet de tirer vers le haut pour soulever l'araire, que ce soit pour en empêcher un trop grand enfoncement ou pour le retirer du sol à la fin de la raie.

21 Pour cette raison, à la différence de la classification suggérée par Gupta et Saraswati et reprise dans le présent volume (cf. Dollfus et al.), il me semble judicieux de dissocier ces araires du Teraï «à mancheron accolé » du manche-sep (d'une seule pièce ou «à mancheron rapporté ») des moyennes et basses montagnes, qu'il faudrait, au contraire, rapprocher de l'araire tibétain et même, à l'instar de Hopfen (1969), du dental. Les premiers conviennent à des sols plus fins, où l'on ne veut pas trop enfoncer le sep, et à un climat plus humide ; les autres à des sols plus grossiers, plus difficiles à pénétrer, et à des climats généralement plus secs. Ce rapprochement me semble également justifié par une plus grande similitude des formes.

Dans les moyennes vallées du versant sud de la grande chaîne, ce sont surtout les versants qui sont cultivés et les pentes sont souvent très fortes. La valeur la plus élevée des pentes cultivées varie d'une région et même d'une vallée à l'autre, en fonction de la pression démographique, mais en général, elle ne dépasse pas $40^{\circ}$. Ces versants sont organisés en systèmes de terrasses plus ou moins horizontales, dont la construction s'étend sur plusieurs années. Celle-ci commence par le labour des pentes dont on a coupé les arbres sans en arracher les souches; les labours successifs, puis des travaux de terrassement, accentuent progressivement les petites inégalités de ces pentes qui, lorsqu'elles sont très fortes, ne sont nettoyées des souches que quand il n'y a plus de danger d'arrachement des sols par le ravinement. On laboure donc durant de longues années sur des champs en pente et l'on a besoin d'un araire très maniable et robuste.

Techniques \& Culture, $37 \mid 2001$ 

sep, on préfère des araires "monobloc » à l'angle d'attaque suffisamment aigu, pour pouvoir les faire rouler (au sens maritime du terme). En effet, le laboureur est parfois contraint de marcher non derrière l'araire mais « en contrebas » et de se pencher pour appuyer sur l'âge, soit parce que la pente est forte soit parce que son champ est trop " court ", comme le disent les paysans de Goljong et Timure (haute Trisuli). Par ailleurs, cette obligation de faire rouler l'araire est peut-être une troisième raison de l'emploi de la douille conique et non de la douille "en pointe de flèche", qui pivote moins facilement, et dont les laboureurs de cette région disent pourtant s'être inspirés il n'y a pas plus de cinq générations ${ }^{12}$. Dans le cas du dental de Jumla, Bajhang et Bajuta, le fait que le manche traverse le sep et son accolement à la chambige lui donnent une grande solidité et une meilleure maniabilité sur les versants en pente.

\section{Les types de labour}

On doit distinguer au moins deux types de labours: le labour de déchaumage et le labour de semailles. Lorsqu'on laboure un champ après une récolte, il faut pénétrer profondément dans un sol tassé par la culture précédente, afin de couper toutes les racines et d'en bien rétablir la structure. Mais, quand on sème, il faut prendre garde à ne pas enfouir trop profondément les semences et à conserver l'humidité du sol, ou encore, dans les rizières, à ne pas trop fatiguer les animaux de trait. Outre l'action sur la longueur de la lanière reliant le timon au joug ou, dans le Teraï, l'utilisation de crans sur le timon, on emploie des seps et des socs distincts pour les différents labours.

Dans les régions de soc à douille, on se sert le plus souvent d'un sep neuf et d'un soc long pour le déchaumage, et d'un vieux sep et d'un soc court ${ }^{13}$ pour le labour de semailles ou de repiquage du riz.

Dans les villages des moyennes montagnes du centre (districts de Nuwakot et Dhading), où l'on peut encore trouver quelques araires neufs d'une seule pièce et à reille, les variations sont nombreuses autour de ces deux thèmes : une reille sur un araire neuf, pour le déchaumage, dans des sols tassés; une douille sur un sep usé ${ }^{14}$ pour les semailles, dans des sols déjà ameublis par un premier labour (fig. 3); ou bien encore une reille neuve sur sep neuf pour le déchaumage et une reille usée sur un sep usé pour les semailles. Philippe Sagant (1976: 58) décrit également l'emploi, dans l'Extrême Est, d'une seule reille, mais de deux seps: l'un, court, pour les sols lourds des rizières inondées et l'autre, long, pour le déchaumage des sols secs. Il ne précise pas si, comme cela est d'usage plus à l'ouest -en particulier en pays Sunuwar (Müller 1984, vol. 1 : 136)-, l'araire pour la boue est un vieil araire de jachère. 


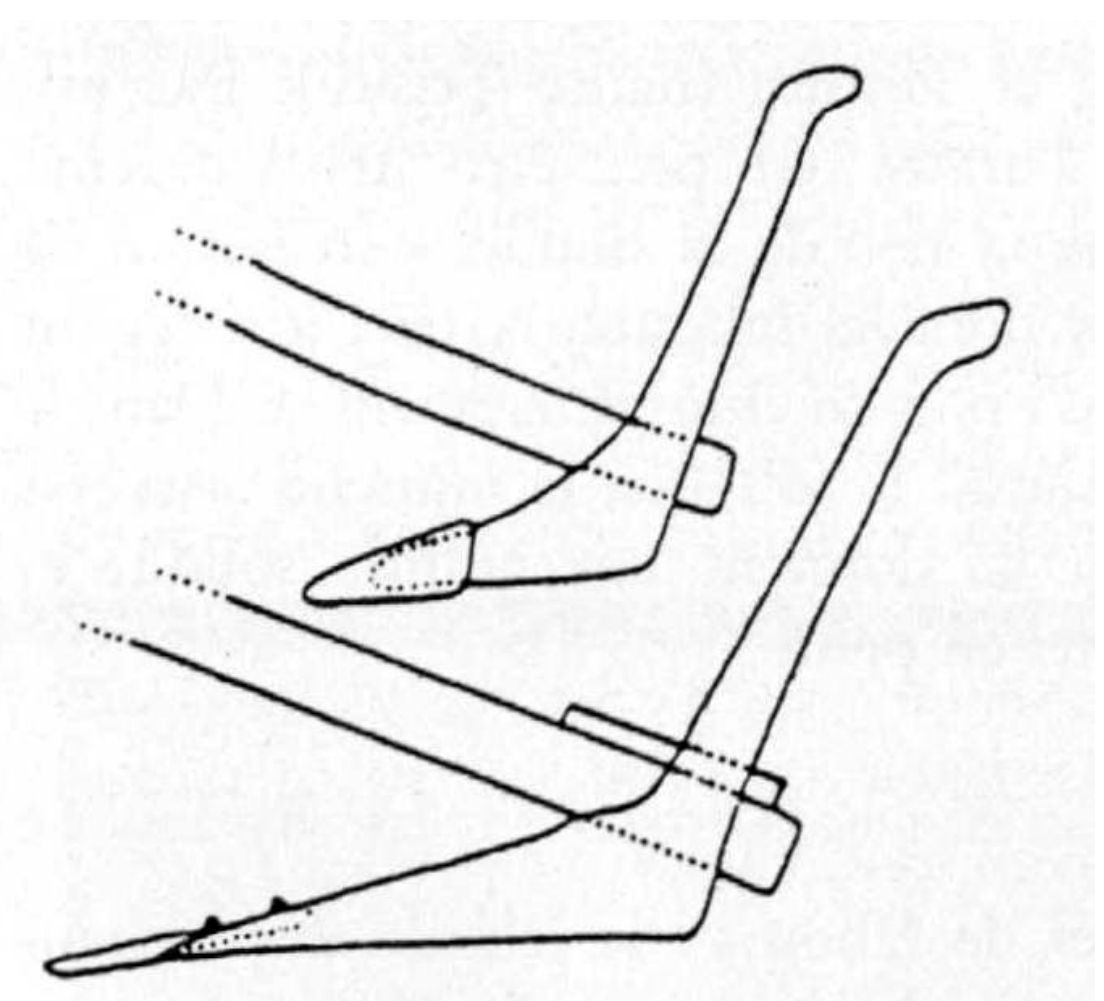

Figure 3. Araires monoblocs des moyennes montagnes (Ankhu Khola); en bas : araire neuf à reille pour labour de déchaumage ; en haut : araire usé à douille pour labour de semailles

\section{La raréfaction du bois}

Toutes les régions ne pâtissent pas de la pénurie de bois dans les mêmes proportions, ni depuis aussi longtemps. La rareté du bois semble s'être toujours fait sentir dans la zone tibétaine, tandis qu'elle est récente sur le versant sud de la grande chaîne. La douille, qui protège la tête du sep, et les ailes, qui en protègent les côtés (et dont nous avons vu les justifications par le type des sols à labourer), permettent la persistance de l'araire manche-sep monobloc dans des régions particulièrement dépourvues de bois, du Mustang ou du Dolpo et, dans une moindre mesure, sur les plus hautes pentes du versant sud. Dans le Haut Mustang par exemple, certains laboureurs qui, il est vrai, n'utilisent leurs araires (en bois de pin) que deux ou trois jours par an, disent les garder pendant vingt ans.

Dans les régions où les seps sont dépourvus d'ailes et où la reille prévaut, soit parce que les sols l'exigent, soit parce que la douille y est inconnue (moyenne Buri Gandaki), le sep n'est pas protégé et s'use très vite. Il doit donc être souvent remplacé. Or, l'araire monobloc du versant sud (en bois de chêne) exige un arbre épais muni d'une grosse branche dans laquelle le manche sera taillé et, en général, on ne peut tailler qu'un seul araire par arbre $\mathrm{a}^{15}$. Il me semble qu'on doit y voir la principale raison de la présence, dans ces régions, du mancheron rapporté sur le sommet du manche-sep (fig. 4) qui semble « remonter » les vallées au fur et à mesure que les ressources en bois s'épuisent. Aux dires mêmes de ses utilisateurs, lesquels déclarent préférer l'araire monobloc, l'araire à mancheron rapporté n'est pas très solide et exige un grand savoir-faire dans les sols durs à travailler. Mais la nécessité de tracer des sillons étroits et profonds empêche l'usage des ailes et douilles, qui économiserait le bois. 
Quant à l'araire-type du Teraï central et oriental (le plus souvent en bois de sal), la technique du mancheron accolé derrière le sep, bien adaptée aux conditions locales, permet également de tailler plusieurs seps dans un seul arbre. Elle économise non seulement du bois, mais du temps. Dans certaines régions du Teraï central, notamment à Janakput où, par endroits, le manque d'eau est aigu, on utilise des reilles en couteau, qui permettent à la fois de tracer de larges sillons et d'économiser le bois (photo 3).

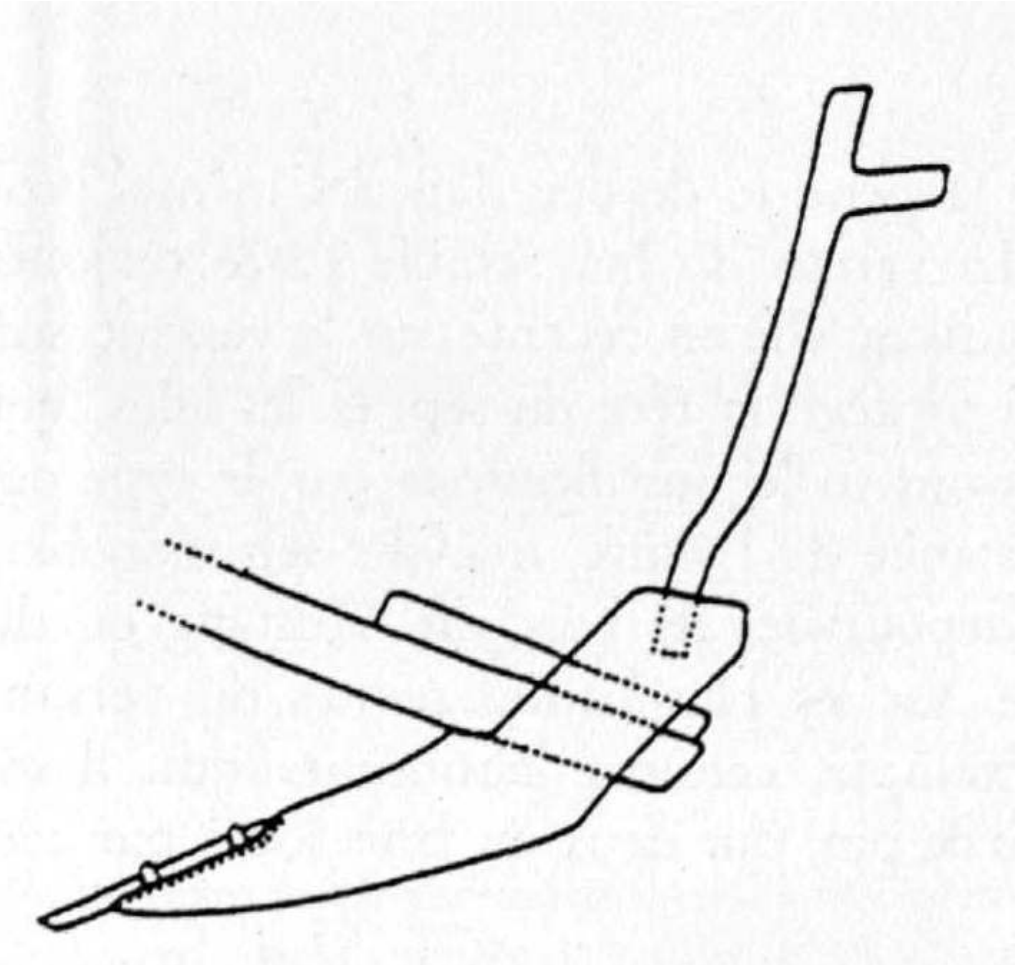

Figure 4. Araire manche-sep à mancheron rapporté (Moyenne Trisuli)

Enfin, selon le point de vue que nous avons adopté ici, l'économie de bois qu'il autorise me semble être la caractéristique principale de l'araire du Teraï de l'Extrême Ouest (ou du haut Kumaon ${ }^{16}$ ). Dans les sols difficiles à travailler de ces régions, la jonction de la tête et du corps du sep est soumise à rude épreuve. Néanmoins, il s'agit du seul araire ne comportant que des pièces droites (photo 1). Si sa fabrication demande moins de bois et moins de manipulations, elle requiert à l'inverse un grand savoir-faire de l'artisan, qui doit être capable de tailler des tenons et des mortaises quadrangulaires. Il s'agit sans doute, si l'on suit les critères d'Haudricourt, de l'araire le plus élaboré de toute la région, malgré son manque apparent d'adaptation aux conditions des labours. Pour ces raisons, mais aussi de par sa forme, lui non plus ne me semble pas pouvoir être associé à l'araire tibétain dans la classification proposée ici.

\section{Les savoir-faire et les coûts}

Tout ce qui a été dit jusqu'ici doit être tempéré par des considérations qui ne sont plus directement liées à l'outil lui-même ou à ses conditions d'utilisation, mais aux caractéristiques, plus individuelles, des systèmes de production ou des exploitations. 

fixer des ailes. S'ils veulent conserver les larges araires monoblocs que la qualité de
leurs champs semble exiger, ils doivent se contenter d'arbres dont l'angle des branches
n'est pas adéquat. L'angle que fait le manche avec le sep est trop obtus et force presque le laboureur à se coucher sur l'araire (fig. 5).

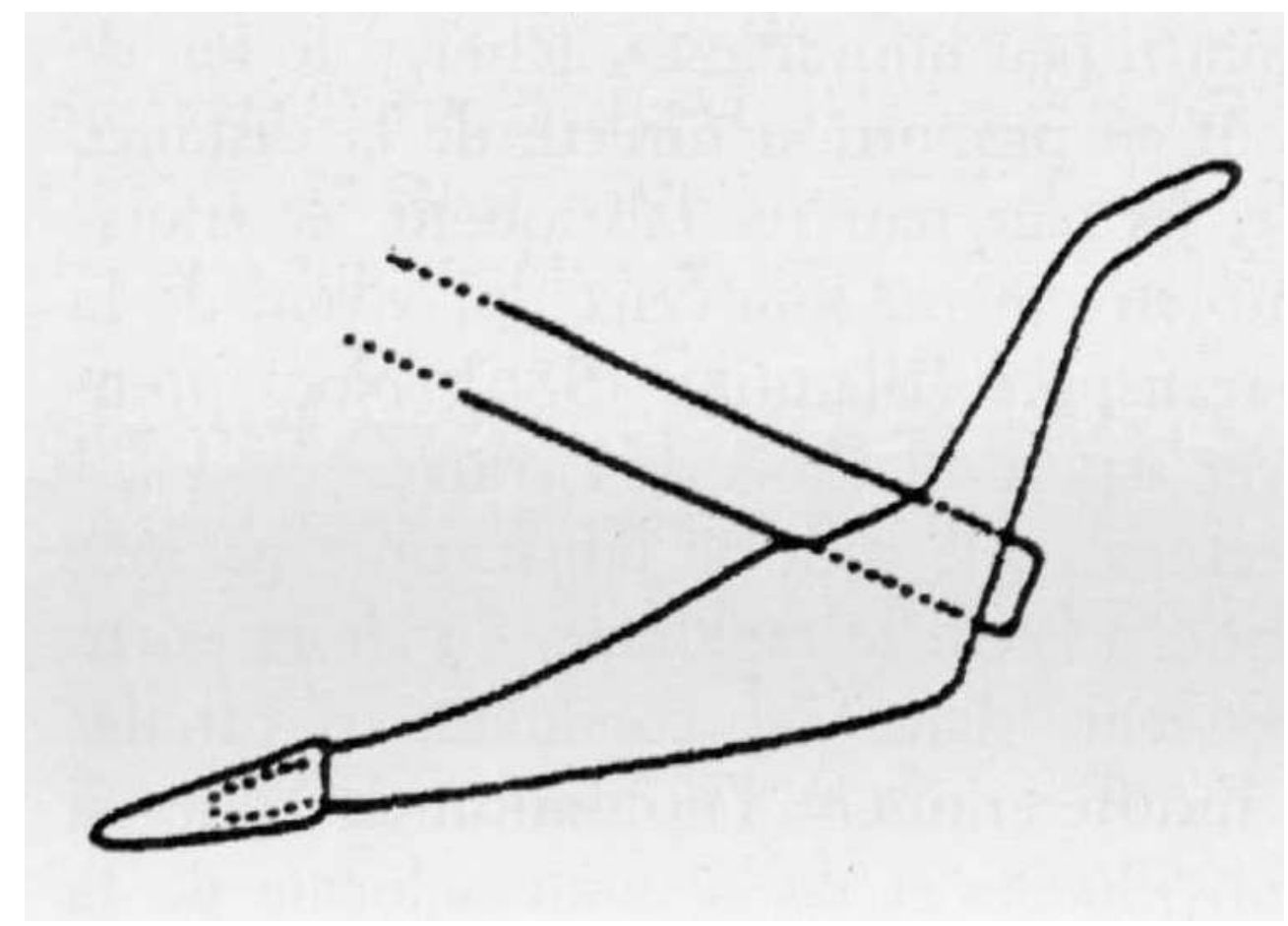

Figure 5. Araire manche-sep à douille de Gatlang, à manche très court et incliné : l'arbre choisi pour le tailler n'était pas adéquat

Si l'on suit Haudricourt, l'araire manche-sep de l'ouest du Népal et du haut Kumaon est plus « moderne » en ce que l'ajustement des pièces y est plus méticuleux et demande un plus grand savoir-faire de menuiserie. L'avantage en est que toutes ses pièces longues sont rectilignes, ce qui permet des économies de bois. Tous ne savent pas fabriquer la tête de sep et son ajustement au manche doit souvent être renforcé par des ligatures, comme au temps des premiers araires. Notons néanmoins que ces ligatures permettent d'élargir le sep et de prévenir une usure trop rapide.

Quant au manche-sep à mancheron accolé, la nécessité de faire varier l'angle d'incidence du sep par des cales au niveau du timon rendrait, même si la technique en était connue, l'utilisation d'un genou impossible. C'est pourquoi ces araires sont parfois 
consolidés par des ficelles qui relient le manche au timon. Ici non plus, les tenons et les mortaises ne sont pas ajustés au plus près (photo 4).

Le contact entre les régions à reille et les régions à douille est intéressant en ce qu'il se fait souvent sur un même versant, à l'intérieur d'un village où les contraintes citées ne dictent pas un choix précis. Il semble alors que cette préférence soit surtout guidée par le savoir-faire et l'habitude et, nous le verrons, par le coût. Sur les versants où l'abondance de la forêt, l'humidité et la nature des sols ne sont pas déterminantes, une des raisons souvent invoquées pour justifier le choix de la reille ou de la douille est la présence de rochers dans lesquels les socs viennent se coincer. Tandis que certains laboureurs disent préférer la reille, fichée dans le sep et maintenue par des crochets, que l'on ne risque pas de perdre lorsqu'on dégage l'araire en le tirant vers l'arrière (ou quand on le soulève en fin de raie), d'autres, au contraire, disent préférer la douille parce qu'on risque moins de casser le sep lorsque le soc se coince. Les uns savent ne pas perdre la douille, les autres ne pas casser le sep équipé d'une reille, chacun vantant évidemment son propre système.

L'économie du Népal est encore très peu monétarisée et acheter un bien n'équivaut pas à le produire soi-même, en termes de coût réel. De même, dans un pays où les routes sont si peu nombreuses, acheter le fer de son soc au village en renchérit le coût en proportion directe de la distance au «bazaar ${ }^{17}$. De façon générale, les plus pauvres fabriquent et transportent ce dont ils ont besoin. Nombreux même sont ceux qui vivent de la valeur ajoutée à un bien par son transport (Blamont 1986). Nous prendrons deux exemples pour illustrer cet aspect du choix de l'araire.

Pas plus que le savoir-faire, le temps ou le coût de fabrication ne sont des facteurs d'explication à long terme, à l'échelle régionale, du choix entre la reille et la douille ; mais ils peuvent, dans des conditions naturelles «neutres ", c'est-à-dire permettant indifféremment l'utilisation de l'une ou l'autre, rendre compte d'options individuelles et de la position locale de la limite entre les deux types de socs. Au village de Jarlang dans l'Ankhu Khola (district de Dhading), par exemple, les dépenses entraînées par les deux socs sont comparables si le laboureur achète seps et socs. Mais s'il fabrique son sep lui-même, la reille (phalli) est plus avantageuse. En effet, pour le même prix (matière première et façon), une douille (phalla) a une durée de vie d'un an, contre deux ans pour une reille; un sep équipé d'une douille dure trois ans et un sep équipé d'une reille un an, pour le même temps de travail (3 jours), effectué, bien sûr, en saison creuse. Les coûts annuels en numéraire sont donc moitié moindres pour un araire à reille si l'on fabrique soi-même son sep avec, toutefois, des dépenses en travail trois fois plus importantes et un sep moins solide et moins maniable.

Cette concurrence entre un coût en travail et un coût en numéraire, donc en définitive, en produits agricoles, est également à l'œuvre dans le cas des reilles en bois qui équipent certains des dentals à Jumla (Khalanga, Hatsija...). La raison le plus souvent invoquée est l'éloignement des marchés (dix jours pour un aller-retour au bazaar le plus proche, Surkhet) où, par ailleurs, les paysans jugent le prix du fer élevé pour leurs faibles ressources. Mais depuis l'ouverture du parc national de Rara, l'accès aux ressources ligneuses est très strictement réglementé. Il devient donc de plus en plus difficile de se procurer les grandes quantités de bois nécessaires (un agriculteur use parfois jusqu'à 80 socs par an). Les habitants du village de Malika $(2620 \mathrm{~m})$, proche du parc, doivent aujourd'hui acheter le fer de leurs socs (des reilles en baguette) soit lors de leurs migrations hivernales en Inde, à Nepalganj, en même temps que sucre, huile, 
vêtements..., qu'ils échangeront dans les villages alentour contre chèvres, moutons, produits agricoles..., soit à l'occasion de leurs trois ou quatre visites annuelles à Surkhet où leur monnaie d'échange est alors le haricot rouge ou noir ( $3 \mathrm{~kg}$ de haricots pour $1 \mathrm{~kg}$ de fer, soit deux socs).

\section{La rencontre des contraintes: quelques types d'araires}

41 La classification régionale proposée ici repose sur le principe même qui a guidé l'analyse précédente: on suppose que les cultivateurs ont visité des régions où ils ont observé des outils différents des leurs, qu'ils ont pu ainsi adapter leurs araires aux diverses contraintes (milieu, savoir-faire, coûts...) ; il n'est donc pas déraisonnable de diviser ceux-ci en types régionaux correspondant à ces contraintes. Cette classification permet également -et surtout- de placer les araires dans des aires plus vastes de localisation des différents types.

\section{Les régions arides}

«[...] il n'est pas possible de conclure à une forme très archaïque de l'araire manche-sep, du moins en ce qui concerne le bâti lui-même de l'araire : le corps de l'instrument est percé d'une mortaise quadrangulaire dans laquelle s'insère le timon, soigneusement ajusté, ce qui suppose des outils de métal et une technique perfectionnée de la charpenterie. L'instrument ne comprend ni ficelage ni étançon, les pièces sont solidement emboîtées les unes dans les autres » (Haudricourt 1986 : 263, à propos des araires du sous-continent indien).

Le manche-sep monobloc à ailes et genou, à sep plus ou moins oblique des régions « tibétaines »

Cet araire, qu'on rencontre notamment au Mustang et au Dolpo, s'apparente aux araires de l'Afghanistan : le mancheron et le sep sont d'une seule pièce, formant un arc de cercle (fig. 1; Dollfus et al: fig. 2.) ; des ailes (signalées par Haudricourt 1986: 262) flanquent le sep ; un genou (venu d'Asie Occidentale -Mésopotamie- via le Sin Kiang, Haudricourt 1986:260) reliant le mancheron au timon consolide l'ensemble et le soc, en fer, est une douille (thunsen) qui enveloppe l'extrémité du sep et se termine en pointe de flèche. Cet araire est particulièrement adapté aux deux grandes contraintes du milieu : les seps sont élargis, pour bien travailler le sol, et protégés de l'usure par des ailes qui sont de petites pièces de bois, parfois des branches assez fines que l'on peut changer souvent et qui assurent une longévité plus grande à l'araire; de la même manière, la forme du soc en pointe de flèche permet un bon travail du sol et une protection efficace du sep. Enfin, la douille en «fer de lance » et le large sep, plus ou moins horizontal selon la grossièreté des sols, répondent particulièrement bien aux exigences du labour dans des sols irrigués, mais où l'eau est rare, et à la fabrication de billons. Le genou, adapté à ces araires larges et difficiles à manier, témoigne à la fois de l'ancienneté et de l'origine tibétaine de cet araire. Soulignons néanmoins que ce genou empêche de faire varier l'angle timon-sep et donc, de contrôler l'angle d'incidence dans le sol, en fonction du type de labour. Ici aussi on utilise un araire neuf pour le déchaumage et un araire usé pour les semailles. 


\begin{tabular}{|l|c|c|c|c|c|c|}
\hline & \multicolumn{3}{|c|}{ Haut Mustang central (Nhenyol) } & \multicolumn{2}{c|}{ nord (Chungjung) } \\
\hline & \multicolumn{2}{|c|}{ longueur } & \multicolumn{2}{c|}{ largeur } & longueur & largeur \\
\hline & neuf & vieux & neuf & vieux & & \\
\hline manche jusqu'au timon & 56 & 42 & 14 & 10,5 & 61 & 7 \\
\hline soc (flèche) & $21(14)$ & $17,5(7)$ & $(14)$ & $(10,5)$ & $21(15,5)$ & $(14)$ \\
\hline sep & 38,5 & 35 & 13,9 & 13,8 & 53 & 20 \\
\hline manche $+\operatorname{sep}+\operatorname{soc}$ & 112 & 89 & & & & \\
\hline timon & 227 & 224 & 24,8 & 32,9 & 210 & \\
\hline aile & 14 & 14 & 3 & 2 & 28 & 4 \\
\hline genou & 14 & 14 & 0,14 & 0 & 30 & \\
\hline poignée & 21 & & & & & \\
\hline
\end{tabular}

Tableau 1. Quelques dimensions d'araires du Haut Mustang (en cm) plus sec. Bien que nous ayons choisi de les classer dans la même catégorie, les araires de ces régions sont de formes très différentes, puisqu'il s'agit d'une part d'un dental et d'autre part, d'un manche-sep. Néanmoins, ils ont été adaptés suivant les mêmes principes aux climats plus ou moins secs. 


\section{L'araire dental de l'Extrême Ouest} des moyennes montagnes du bassin versant de la Bheri et de ses affluents, à l'extrême ouest du Népal : Jumla, Bajura, Bajhang... Nous sommes ici à la pointe orientale d'une aire de répartition qui englobe toute l'Asie Occidentale, depuis Chypre et la Crète, l'Arménie, l'Irak et l'Iran, l'Afghanistan, jusqu'au bas Kumaon voisin. On retrouve la forme toute particulière des araires de la Transcaucasie, de l'Arménie, de l'Iran, du Daghestan et du Khûmen : le mancheron est fixé sur le sep au pied de la chambige; le talon, qui déborde vers l'arrière, permet l'action du pied. À Jumla, cet araire est souvent équipé d'une reille en bois.

\section{L'araire monobloc à douille conique, sans ailes et sans genou}

On le trouve sur le versant sud de la Grande chaîne, au centre du Népal (bassin versant de la Trisuli et de ses affluents). Si sa forme s'apparente à celle de l'araire tibétain, il en diffère par sa douille conique ${ }^{21}$ qui, aux dires des paysans, pénètre mieux dans des sols plus grossiers, en se coinçant moins facilement sous les gros blocs des sols sur versants d'éboulement. Son sep est souvent long et large et il convient à des champs de versants en pente plus ou moins enterrassés, qui requièrent un araire solide et maniable. Ses dimensions varient de $60 \mathrm{~cm}$ (pour un sep neuf utilisé lots du déchaumage) à 35 (pour un sep usé utilisé lors des semailles). Il est menacé par le manque du bois.

\section{L'araire à mancheron rapporté et à reille, à sep étroit, sans genou ni ailes}

On l'observe dans tout le Mahabharat parbatya central et oriental, depuis le bassin versant inférieur de la Kali Gandaki jusqu'à celui de la Sapti et de ses affluents, en passant par les pays tamang, en aval des régions à araire monobloc à douille; il s'agit d'un araire à " mancheron rapporté » sur le sommet du sep, c'est-à-dire en deux pièces, " deux pièces n['en] formant en réalité qu'une " (Haudricourt 1986:253). Sa longueur peut atteindre $70 \mathrm{~cm}$. Le sep en est étroit ${ }^{22}$ et n'est pas muni d'ailes : le climat est humide et les sols sont en général assez fins; la densité des sillons doit y être plus grande. Ce sont les femmes qui assurent l'émiettage des mottes à l'aide de masses en bois munies d'un long manche. Enfin, l'angle du sep avec le timon est très obtus, ce qui transforme presque l'ensemble manche-sep en un arc de cercle dont le centre serait le point d'attache du timon sur le joug. Cela permet, lorsque les zébus tirent et quand on appuie sur le manche, de faire porter la pression non sur l'ensemble de la semelle du sep mais sur le soc et donc, de l'enfoncer, ou, à l'inverse, de le soulever. Il s'agit peutêtre d'une adaptation de l'araire monobloc au manque de bois: en effet, puisqu'on n'a plus besoin de tailler le manche dans une branche faisant un angle précis avec le tronc, on peut tailler plusieurs seps dans un seul arbre.

\section{L'araire manche-sep monobloc à reille}

Très rare aujourd'hui, il est parfois associé à l'araire monobloc à douille conique mais sans ailes dans certains des villages de contact entre les régions où l'on ne trouve que ce dernier et celles où l'on emploie le manche-sep à mancheron rapporté à reille ; d'une longueur de $70 \mathrm{~cm}$, il sert aux labours de déchaumage (photo 5$)^{23}$. Selon les agriculteurs

Techniques \& Culture, 37| 2001 
des villages de Gatlang et de Goljong, le soc à douille serait une innovation récente (pas plus de cinq générations) en provenance du Tibet $^{24}$; cet araire monobloc à reille pourrait donc bien être l'araire le plus ancien des moyens pays de l'ouest, centre et est népalais ${ }^{25} \mathrm{La}$ raréfaction des ressources en bois, d'une part, l'introduction de la douille depuis le Tibet, d'autre part, seraient des raisons plausibles de l'évolution de cet outil vers l'araire monobloc à douille des régions les plus élevées de cette frange altitudinale et vers l'araire à reille et mancheron rapporté des régions les plus basses.

\section{Les formes intermédiaires}

51 . Araire avec ailes (très proche de l'araire " tibétain »), sans genou. On le trouve dans la haute Trisuli, dans les villages les plus élevés des régions de l'araire monobloc à douille conique mais sans ailes, au contact de régions ribéraines avec lesquelles s'est établi un courant d'échanges.

52 . Araire à mancheron rapporté et à douille conique. On le trouve, mais très rarement, dans les villages des étages inférieurs des régions de l'araire monobloc à douille conique, au contact des régions de l'araire à reille, où il est utilisé pour les labours de semailles. Il est en effet particulièremenr fragile.

\section{Les régions de plaine}

53 Ici aussi, on note une différence entre les araires de l'est et ceux de l'extrême ouest du pays, région plus sèche et dont les sols sont parfois plus grossiers.

\section{L'araire manche-sep à mancheron accolé}

On le rencontre dans tout le Teraï du centre et de l'est, mais aussi dans le nord du Bihar ${ }^{26}$ tout proche (Haudricourt 1986 : 261, fig. 118). Le manche, fin et plus ou moins long, est accolé au sep, plus large, par le timon qui en assure la cohésion (photos 3 et 4) ${ }^{27}$. Ce manche semble surtout adapté à un mouvement de levage de l'araire en fin de raie ; il peut aussi empêcher l'instrument de s'enfoncer trop dans des sols argileux et facilement pénétrables. Cet araire est donc bien différent de l'araire à mancheron rapporté, dont la fonction semble être surtout d'enfoncer l'araire dans des sols plus grossiers. Au centre et à l'est, la profondeur du labour est, en outre, généralement contrôlée grâce à des crans dans le timon qui permettent de l'allonger ou de le raccourcir et de changer l'angle d'incidence du sep. Ailleurs, c'est plutôt la longueur de la courroie qui relie le timon au joug qui règle cette profondeur.

\section{Le manche-sep à tête de sep rapportée}

On le trouve dans les bas pays de l'extrême ouest du Népal (bassin de Surkhet), ainsi que dans le haut Kumaon voisin (photo 1). Sa durée de vie dépend bien sûr des superficies à labourer et du nombre de labours, qui oscille entre deux et trois ; mais en général, elle n'excède pas deux ou trois ans. La largeur du sep varie d'une dizaine de centimètres sur les sols les plus humides et grossiers du sud du bassin de Surkhet (où il est équipé d'une reille traversant le manche) à une trentaine de $\mathrm{cm}$ sur les terrasses fluviales les plus sèches. 


\section{Histoire et évolutions ?}

dental de l'Extrême Ouest et les manche-seps du Centre et de l'Est, qui semble cependant correspondre à des limites de bassins versants, donc d'aires d'échanges. Néanmoins, si l'on considère les régions entourant le Népal, on note une similarité certaine entre les araires en usage de part et d'autre des frontières, et l'on serait alors tenté de conclure à une conjugaison d'héritages et d'influences d'aires culturelles et techniques ( $c f$. Dollfus et al., ce volume). En effet, les Népalais voyagent beaucoup et n'hésitent pas à améliorer leurs instruments en s'inspirant de ce qu'ils ont observé ailleurs, ou même à adopter et adapter de nouveaux outils. Ainsi, l'ubiquité de l'araire à mancheron accolé, à l'instar de l'araire « du Bihar » dans le Teraï du Centre et de l'Est, zone de colonisation récente depuis les moyennes montagnes, prouve qu'ils peuvent abandonner un araire (celui de leur région d'origine) quand celui-ci ne convient pas aux conditions de la région dans laquelle ils viennent s'installer; et cela d'autant plus que les gestes du labour ne sont pas très différents. C'est pourquoi, comme pour la limite séparant les régions de la douille de celles de la reille, les raisons de l'emplacement exact de la démarcation entre les différentes aires de répartition des araires doivent plu tôt être cherchées parmi les déterminants que nous venons de passer en revue. Plus encore que l'évolution d'un araire monobloc à reille vers un araire monobloc à douille ou vers un araire à manche rapporté à reille, qui reste de l'ordre de l'hypothèse, l'exemple du nombre d'animaux de labour et du type d'attelage nous permettra de repérer quelques manifestations récentes de cette recherche -qui semble constante- de l'outil le plus adéquat et de l'adaptabilité plus ou moins grande et plus ou moins rapide des araires à de nouvelles conditions.

En 1986, la Banque de Développement Agricole du Népal, afin de trouver une solution au manque de bois, tenta d'introduire dans le Teraï central une charrue, importée de Malaisie, de type chinois avec timon recourbé et palonnier, attelée à un seul animal. Mais, lors de la démonstration de l'outil -organisée en grande pompe- le zébu, malgré sa taille, se mit très vite " en grève » en se couchant ; il refusait d'être le seul à tirer un objet si pesant qui fut donc rangé au musée des innovations avortées, parce que trop différentes des outils locaux. Le dressage des zébus au labour solitaire risque d'être difficile : pour la plupart, ils semblent peu robusres. Lorsque l'on attelle ensemble deux zébus de forces différentes, il n'est pas rare de voir le plus fort donner des coups de corne à son jeune " collègue " pour l'inciter à " mettre un bon coup de collier ».

Au Tibet, à l'inverse, c'est pour pallier le manque de yaks que de nombreux agriculteurs $\mathrm{du}$ sud de Lhassa ont adopté une charrue à palonnier ${ }^{28}$ venue de Chine, qui semble moins appropriée à leurs sols que l'ancien manche-sep monobloc à ailes et à douille, lequel, ne les retournant pas, ne les assèche pas autant. Ici non plus, on n'a pas su encore dissocier le type d'outil (araire ou charrue) de l'attelage. Autant, dans le cas précédent, il s'agissait de garder le type d'attelage mais de passer de l'araire à la charrue, autant il s'agirait ici de garder l'araire et de changer l'attelage.

Cette hybridation a néanmoins eu lieu depuis quelques années dans le Teraï central (Janakpur), où l'on utilise une charrue très sommaire qui est en fait un instrument quadrangulaire à sep horizontal et soc dissymétrique en fer, agrandi en versoir fixe versant à gauche (apparentant davantage l'outil à une pelle trânée qu'à un araire), sans coutre. Les agriculteurs disent l'avoir trouvée dans les régions indiennes avoisinantes 
et affirment qu'elle n'a aucun rapport avec le manche-sep qu'ils employaient auparavant, sinon que le timon, identique, est directement relié à un joug de garrot et qu'elle n'a qu'un mancheron. Le labour ne se fait plus alors en raies parallèles mais en spirale centripète, ce que certains pratiquaient déjà. Nonobstant l'absence de palonnier, cette charrue me paraît être, par sa forme et celle de son versoir, d'origine chinoise. On peut néanmoins s'étonner, non de l'absence de coutre pour «couper » le sol $^{29}$, mais de son soc peu pointu, triangle très allongé vers l'avant. Il paraît s'expliquer par la nature des sols, alluviaux fins, irrigués, très tendres.

Ce qui pourrait expliquer l'adoption de cet instrument est précisément l'absence de palonnier, et donc, le maintien de l'attelage de deux zébus. Ses utilisateurs ont, eux, su opérer la disjonction du palonnier et de la charrue, du timon et de l'araire, pour produire un hybride, une charrue à long timon sans palonnier.

Bien que les innovations ne se fassent pas toujours immédiatement dans les formes les plus adaptées à l'ensemble des contraintes, il faut voir dans ces exemples des preuves de la volonté et, pour certains, de la capacité des laboureurs de répondre à l'évolution des conditions des labours par l'emprunt de nouveaux outils ou par l'adaptation des anciens.

62 La classification des araires du Népal, ici ébauchée, est fondée sur l'hypothèse d'une adaptation des formes aux différentes contraintes: la nature des champs et leur localisation influencent la forme et la taille du sep ainsi que le choix du soc (douille en fer de flèche ou conique, reille), tandis que la rareté du bois implique des adaptations qui vont de l'ajout d'ailes, dans les régions sèches, à la technique de la tête de sep ou du manche rapporté ou accolé, dans des milieux plus humides. Nous distinguons donc trois " tendances ${ }^{30}$ " en fonction d'une classification des milieux et des contraintes imposées par ces milieux, d'abord, sur la technique du labour et sur la forme générale de l'instrument, puis sur sa fabrication et sur le nombre de ses pièces, que nous considérons plutôt, toujours selon la terminologie de Leroi-Gourhan, comme des « degrés du fait ». On aboutit ainsi aux deux catégories suivantes.

63 1) L'araire des hauts pays secs et de pentes peu accentuées, de culture tibétaine : un manche-sep monobloc, à ailes et genou, à douille en fer de lance.

64 2) Les araires des moyennes montagnes plus ou moins humides, adaptés à des champs de versants aux pentes plus ou moins fortes. Il nous faut distinguer l'ouest de l'est du Népal; l'est: un araire manche-sep des moyennes montagnes (sans ailes ni genou), dont nous supposons qu'une forme originelle (araire monobloc à reille) aurait évolué vers un araire monobloc à douille conique dans les villages amont, et un araire à mancheron rapporté sur le sommet du sep dans les villages de l'aval ; l'ouest: l'araire dental à chambige et reille (en fer ou en bois) des hauts et moyens pays secs.

3) Les araires des plaines du Teraï. Là encore, il faut faire une distinction entre l'est et l'ouest; l'est: un araire manche-sep en deux pièces dont le manche, fin et plus ou moins long, est accolé au sep, plus large, par le timon qui traverse les deux pièces et en assure la cohésion; l'ouest: un araire manche-sep à tête de sep rapportée, particulièrement adapté au manque de bois et à des sols plus ou moins grossiers, mais fragile et exigeant un grand savoir-faire, tant pour sa fabrication que pour son maniement.

66 Cette classification nous semble la plus compatible avec les principes adoptés par Haudricourt, tout en étant pertinente à l'échelle régionale. Elle privilégie les traits 
d'adaptation aux contraintes par rapport aux critères morphologiques et rapproche des formes très différentes en même temps qu'elle distingue des variations fonctionnelles d'une même forme. Les tentatives d'introduction de nouveaux types, avec des bonheurs divers, semblent à la fois justifier les principes de cette classification et relativiser celleci -qui risque bien d'être tout autre dans quelques années.

\section{BIBLIOGRAPHIE}

Blamont, Denis

1983, « Agricultural techniques in High Central Nepal : choice and adaptation to constraints by Tibeto-Birman groups ", $8^{\text {th }}$ European Conference on Modern South Asian Studies. Tällberg (Suède 2-8 juillet).

1986, « Les échanges locaux et régionaux », pp. 167-182, in J.-F. Dobremez (éd.), Les Collines du Népal Central : écosystèmes, structures sociales et systèmes agraires. Paris : INRA.

1987, « Systèmes de production et habitat en pays tamang du centre-ouest du Népal », pp. 41-76, in D. Blamont et G. Toffin (eds), Architecture, milieu et société en Himalaya. Paris : Éditions du CNRS.

Haudricourt, André-Georges et Mariel J.-Brunhes Delamarre

1986, L'homme et la charrue à travers le monde. Lyon : La Manufacture (1 ère édition 1955).

Hopfen H.-J.

1969, « Farm implements for arid and tropical regions », FAO 91.

Leroi-Gourhan, André

1971, Évolution et techniques. I - L'homme et la matière. Paris : Albin Michel (1 1 ère édition 1943).

Millier, Bruno

1984, Terre et paysans du Népal. Le système de production et son évolution dans un village Sunuwar multi-ethnique. Thèse de Doctorat de $3^{\text {ème }}$ cycle. Université de Rouen.

Sagant, Philippe

1976, Le paysan limbu : sa maison et ses champs. Paris : Mouton.

Wiart, Jacques

1983, Écosystème villageois traditionnel en Himalaya népalais : la production forestière suffit-elle aux besoins de la population? Thèse $3^{\text {ème }}$ cycle. Université de Grenoble.

\section{NOTES}

2. L'araire chambige semble être inconnu dans toute la région himalayenne.

3. Pour reprendre la terminologie de Leroi-Gourhan (1971).

4. C'est-à-dire « inventées » ou « empruntées »; faute d'informations exhaustives, nous ne ferons pas la différence.

5. Où les premiers labours ont en général lieu avant la mousson.

6. Ici, la fonction de l'araire est «non seulement de tracer une raie, mais aussi de remuer, d'émietter la terre [...] pour lutter contre la sécheresse [car] l'ameublissement superficiel du sol 
[empêche] l'évaporation de l'eau ", comme le fait remarquer Haudricourt (1986:259) à propos des araires méditerranéens.

7. « Le plus caractéristique » du Pakistan et de l'Inde Septentrionale (Haudricourt 1986 : 261).

8. Comme pour certains sols très secs du district d'Almora dans le Kumaon indien.

9. Sauf mention expresse, le terme de reille est ici employé pour désigner des reilles en forme de «baguette ».

10. Le «fer de flèche" serait, d'après Haudricourt, "la forme de soc la plus répandue actuellement » et connue depuis longtemps : il « ouvre le sol et coupe par ses côtés les racines des herbes » (Pline cité par Haudricourt 1986 : 109). Au Népal, il s'agit de la forme la plus rare.

11. Haudricourt (1986: 94 et 95) le décrit ainsi : « l'espace du sep ou dental compris entre la base du timon et celle du mancheron est destiné à recevoir le pied du laboureur [...]. Le laboureur complète et prolonge ainsi la pression exercée par la main sur le mancheron par tine pression encore plus directe sur les parties travaillantes de l'instrument... Association de gestes liés à la fonction de l'outil et aux conditions géographiques les plus fréquentes dans lesquelles s'exerce le travail du cultivateur : travail en surface dans un terrain sec et pierreux; il faut faire des pieds et des mains pour maintenir l'appareil dans le sol!"».

12. Haudricourt penche généralement pour une antériorité de la reille sur la douille.

13. Il s'agit soit de socs usés, soit de socs que l'on a fabriqués ainsi.

14. Dispositif qui a pour effet une diminution de la distance de la pointe du soc au timon qui déterminerait la profondeur du labour.

15. J. Wiart (1983 : 205-209) explique en outre que l'on coupe parfois deux ou trois arbres avant de trouver une branche assez solide et faisant l'angle requis avec le tronc.

16. On le trouve aussi au Pendjab.

17. À titre d'illustration de ce caractère très contraignant des distances, on peut, bien qu'il n'ait aucun rapport direct avec ce qui nous occupe ici, citer le fait que certains paysans de Bajhang vivent tellement loin du bazaar le plus proche (Chaïnur) que, ne disposant pas d'animaux de bât, ils doivent renoncer à aller y vendre leurs pommes de terre : ils ne pourraient porter que ce qui leur serait nécessaire à se nourrir pendant leur voyage...

18. Effectués soit successivement par le même laboureur, soit simultanément par plusieurs attelages.

19. Phalla à Baragaon (partie inférieure du haut Mustang).

20. 12 roupies valent à peu près 1 franc.

21. La douille est dite thunsen en haute Tsum et phalla sur le versant sud. La reille est dite phalla en Haute Tsum et phalli sur le versant sud.

22. Phénomène très général au Népal : le plus souvent, la largeur des seps décroît avec l'altitude.

23. On le trouve également en Inde, dans le nord de l'Orissa, chez certains Santal vivant dans les régions accidentées les plus reculées.

24. D'après Kamitashi de Barang (Dhading), âgé de 83 ans en 1984, son grand-père utilisait également une douille fer de lance à l'époque où seul le haut du versant cultivé actuel était habité et mis en valeur, comme c'était le cas dans l'ensemble de la région de Rasuwa (Blamont 1987) et des hauts Nuwakot et Dhading.

25. Cela permettrait de rendre compte de sa présence dans la vallée de Katmandou.

26. Où cependant, selon Haudricourt, il est équipé d'une douille. Par ailleurs, son appellation par Haudricourt d'araire "du Bihar» est abusive, car ce type ne se retrouve ni dans la partie orientale et méridionale du Bihar, le Chota Nagpur peuplé de Munda, ni dans l'Orissa tribal (Santal ou Jhuan), pas plus qu'en Andhra Pradesh.

27. Soit le timon traverse le manche, soit c'est l'inverse : cela dépend de la largeur du manche.

28. Franchissant ainsi « la limite très significative qui sépare la zone de la charrue et de l'attelage à palonnier, à un seul animal, de la zone à l'araire à attelage au timon. » (Haudricourt 1986 : 271).

29. Qui « n'apparaît pas en Extrême-Orient. » (Haudricourt 1986 : 282). 
30. Au sens de Leroi-Gourhan (1971: 326) : « un mouvement, dans le milieu intérieur, de prise progressive sur le milieu extérieur »; "la notion de tendance couvre, d'une autre manière, le déterminisme technique $»$.

\section{RÉSUMÉS}

La classification des araires du Népal présentée ici est fondée non tant sur leurs formes que sur leur adaptation à un faisceau de contraintes liées à la nature des sols, aux types de labour, à la disponibilité des matériaux de fabrication et aux savoir-faire locaux. On distinguera ainsi trois grands types: l'araire des hauts pays secs et aux champs irrigués en pentes faibles; les araires des moyens pays humides, adaptés à des champs de versants aux pentes souvent fortes; les araires des plaines du Teraï. Chacune de ces familles est ensuite divisée en sous-types selon la plus ou moins grande humidité des milieux, mais où les critères morphologiques trouvent également leur place.

The present article proposes a classification of the ploughs (ards) in Nepal, based much more on their adaptation to a set of constraints than on their forms. The constraints include the nature of the soils, the types of ploughing, the availability of prime materials and the local know how. Thus three main types have been identified: the ard of the dry highlands where the fields are irrigated and more or less horizontal; the ards of the more humid mid-mountains, where the fields are situated on more or less steep slopes; the ards of the Teraï plains. Each one of these families is then divided into sub-types, depending on the humidity of the climates, but wheremorphological criteria arc also taken into account.

Esta clasificación de los arados de Nepal se funda sobre su adapración respecto a un conjunto de restricciones que depende de la naturaleza de los suelos, de los materiales de fabricación, de los saberes locales -más que sobre sus formas. Así se diferencian tres grandes tipos : el arado de las regiones altas y secas con campos de regadío en pendiente suave; los arados de las regiones medianas y húmedas, adaptados a campos de vertientes, a menudo con pendientes fuertes; los arados de los Ilanos del Terai. A continuación, cada una de estas clases se divide en varias subclases, según la húmedad de los ecosistemas, y que toman igualmente en cuenta los criterios morfológicos.

\section{INDEX}

Mots-clés : adaptation, araire, labour, milieu, reille, sols

\section{AUTEUR}

\section{DENIS BLAMONT}

UPRESA 7005. Parc d'innovation. 5, Boulevard S. Brant. F-67400 Illkirch. denis@lune.u-strasbg.fr 